\title{
Reactive localized $\pi$-radicals on rim-based pentagonal rings: properties and concentration in flames
}

\author{
Angiras Menon ${ }^{\mathrm{a}}$, Jacob Martin ${ }^{\mathrm{a}}$, Gustavo Leon ${ }^{\mathrm{a}}$, Dingyu Hou ${ }^{\mathrm{b}, \mathrm{c}}$, Laura Pascazio ${ }^{\mathrm{a}}$, Xiaoqing You ${ }^{\mathrm{b}, \mathrm{c}}$, Markus Kraft ${ }^{\mathrm{a}, \mathrm{d}, *}$ \\ ${ }^{a}$ Department of Chemical Engineering and Biotechnology, University of Cambridge, West Site, Philippa Fawcett Drive, Cambridge, CB3 0AS, \\ United Kingdom \\ ${ }^{b}$ Center for Combustion Energy, Tsinghua University, Beijing, 100084, China \\ ${ }^{c}$ Key Laboratory for Thermal Science and \\ Power Engineering of Ministry of Education, Tsinghua University, Beijing, 100084, China \\ ${ }^{d}$ School of Chemical and Biomedical Engineering, Nanyang Technological University, Singapore 637459
}

\begin{abstract}
The impact of localized $\pi$-radicals on soot formation is explored by considering their electronic structure and computing their relative concentrations in flame conditions. Electronic structure calculations reveal that the presence of localized $\pi$-radicals on rim-based pentagonal rings is due to aromaticity. We further calculated a complete mechanism for the formation and elimination of the site from hydrogen additions and abstractions. A batch reactor with flame concentrations of $\mathrm{H}^{\bullet}$ and $\mathrm{H}_{2}$ was used to determine the time-dependent concentration of localized $\pi$-radicals. Low temperatures $(<1000 \mathrm{~K})$ favored the fully saturated rim-based pentagonal ring. Soot nucleation temperatures $(1000$ $1500 \mathrm{~K}$ ) give way to unsaturated rim-based pentagons being favored. Localized $\pi$-radicals on rim-based pentagonal rings are found to be in higher concentration than the aryl-type $\sigma$-radical on the rim-based pentagon (mole fractions of $10^{-6}-10^{-7}$ ) in below $<1500 \mathrm{~K}$, consistent with recent experimental observations. Higher temperatures favor the $\sigma$-radical and the concentration of the localized $\pi$-radical on rim-based pentagons becomes negligible. A kinetic Monte Carlo treatment of multiple sites indicates that multiple localized $\pi$-radicals are possible on a single molecule. These results reveal the importance of localized $\pi$-radicals on rim-based pentagonal rings for PAH chemistry leading to formation of soot nanoparticles in flames involving aromatic rim-linked hydrocarbons (ARLH).
\end{abstract}

Keywords:

localized $\pi$-radicals, partially saturated rim-based pentagon, aromaticity, aromatic rim-linked hydrocarbons, soot formation

*Supplementary Material is available for this paper.

Colloquium submitted for: Gas-phase Reaction Kinetics

Method of Determination: Method 2

Total Word Count: 4247 words, 8.0 pages

\footnotetext{
*Corresponding author

Email address: mk306@cam.ac.uk (Markus Kraft)
} 


\section{Introduction}

Soot nanoparticles invade our bodies and damage almost every organ [1]. Within the atmosphere they absorb large amounts of infrared light, contributing to global heating on nearly the same scale as carbon dioxide $[2,3]$. Fundamental insights into this process have remained elusive, but recent experimental techniques such as high resolution atomic force microscopy (HRAFM) [4] and tunable photoionisation time of flight mass spectrometry (PI-TOF MS) [5] are beginning to uncover new reactive aromatic soot precursors that lead to soot formation and its potential elimination. We recently mapped the reactivity of the observed edges using electronic structure theory with reactivity indices [6]. We found localized $\pi$-radicals on rim-based pentagonal rings that are partially saturated/protonated (previously explored computationally for edge rearrangements [7]). This enables complexes that are both $\pi$-stacked and covalently bonded, forming a rim-bond on the face of the aromatic species. We call these complexes aromatic rim-linked hydrocarbons (ARLH). The importance of localized $\pi$-radicals in soot formation was first introduced by Wang at the 2010 International Symposium on Combustion [8], where he considered a separate localization mechanism based on biradical systems such as acenes $[8,9]$.

HR-AFM of aromatic soot precursors showed the presence of these partially saturated sites as well as fully saturated rim-based pentagons [4]. This would suggest that a partial equilibrium exists between rim-based pentagonal rings and hydrogen radicals in flames, potentially leading to significant concentrations of reactive localized $\pi$-radicals. The concentration of another radical - the $\sigma$-radical - was computed by Howard in 1991 [10], where the edges of these aromatic species were considered to be in partial equilibrium with $\mathrm{H}^{\bullet}$ and $\mathrm{H}_{2}$ in the flame. On average, 2-3 $\sigma$-radicals were found to be present in each $\approx 670 \mathrm{Da}$ aromatic species [10]. However, no similar analysis exists for partially saturated rim-based pentagonal rings.

In this paper, we first explore the localization of $\pi$ radicals. A reaction mechanism and rate constants are then calculated for partially saturated rim-based pentagonal rings. Batch reactor simulations using kinetic equations and Monte Carlo methodologies are then used to explore the concentration of these reactive $\pi$-radicals in flames.

\section{Methodology}

Geometry optimizations and vibrational frequency calculations were performed using the hybrid density functional B3LYP and the 6-311G(d,p) basis set for all chemical species and transition states. Transition states were confirmed to have one imaginary frequency corresponding to the reaction in question. The energies of all major species and transition states were further refined using the composite CBS-QB3 method. All quantum chemistry calculations were performed using the Gaussian 16 program [11].

The rate constants of all reactions with a well-defined transition state and barrier were estimated using conventional transition state theory as implemented in the Arkane package within Reaction Mechanism Generator [12]. Partition functions were evaluated using the rigid rotor harmonic oscillator approximation and quantum tunneling corrections were applied through the Wigner correction [13]. Eckart tunneling also gave very similar results. For the barrierless hydrogen-PAH radical recombinations, two different approaches were used to estimate the rate constant. First, RRKM theory with a simple Gorin Model was used as implemented in Unimol [14]. The potential energy as a function of fragment separation was fitted to a Morse potential and the energies of the separated fragments were taken from the CBS-QB3 energies. However, since the PAH fragment in this case is large, the Gorin model is less reliable. Hence, the rate constants for the barrierless channels were also estimated using variational transition state theory (VTST) [15] as implemented in ktools within Multiwell [16]. Trial transition state geometries and vibrations were taken from potential energy surface scans of the PAH-H bond using B3LYP/6-311G(d,p). The energy and momentum maxima and grain sizes were taken from [17]. Ultimately, the VTST rate constants were used as the Gorin model appeared to greatly overestimate the recombination reaction rate constants and were deemed unreliable . Further details on the rate calculations are provided in the supplemental information.

Kinetic simulations were performed in a 0-D isothermal reactor using Cantera [18] at a pressure of 1 atmosphere and initial mole fractions of $10^{-7}, 0.001$, and 0.1 for acecoronene, $\mathrm{H}$, and $\mathrm{H}_{2}$, respectively, with the rest as $\mathrm{N}_{2}$. These are representatives of typical concentrations of these species in flames [19]. The rate constants for the reactions of the five-membered ring in acenaphthalene were used, as it is expected that these will be very similar to those for the five-membered ring in acecoronene. Justification is provided in the supplementary information. In addition, two kinetic Monte Carlo (KMC) simulations were conducted to study the effect of multiple five-membered rings on the formation of localized $\pi$-radical sites . Coronenes with two and three rim based five-member rings were selected as the 
starting PAHs for each KMC simulation. Only the 18 reactions involving the localized $\pi$-radical were included as jump processes. The initial concentrations and pressure were the same as for the 0-D kinetic simulations with a temperature of $1000 \mathrm{~K}$. An ensemble of 100,000 PAHs was used per simulation to achieve statistical significance. Further details on the KMC model are provided in the supplemental information.

\section{Results and discussion}

\subsection{Localization of $\pi$-radicals}

Localization of $\pi$-radicals is driven by aromaticity. We will highlight this by considering the spin density interpreted through a Clar analysis for $\pi$-radical doublet spin states.

Figure 1 shows the spin density isosurface as well as the value of the spin density for the most spin-rich site. The phenylmethyl radical [Fig. 1 a)] shows the most significant spin density on the methyl site. A Clar analysis would suggest that the phenyl ring contains an aromatic sextet ("six $\pi$-electrons localized in a single benzene-like ring separated from adjacent rings by formal CC single bonds" $[20,21])$ with the radical residing solely on the methyl group. Spin density is seen within the aromatic ring suggesting other minor Clar resonance structures, however, the Clar structure shown is found to dominate suggesting that the aromaticity of the ring is maximized when the $\pi$-radical is localized to the methyl site. Similarly with the fluorenyl radical [Fig. 1 b)], the major Clar structure suggests two sextets with the radical localized to the pentagonal ring, which is seen in the spin density. A Clar analysis of the localized- $\pi$ radical [Fig. $1 \mathrm{c}$ )], the focus of this paper, would suggest a traveling sextet across the two six-membered rings with the radical localized on the unsaturated pentagonal ring site. The spin density suggests that some minor delocalization is occurring to the $\beta$-site from the primary radical site on the aromatic edge due to minor Clar structures [6]. The fact that this site is not significantly delocalized past the $\beta$-site is seen from the lack of change in the spin density after enlarging the aromatic network [Fig. 1 d)]. Furthermore, no significant change is seen for the same $\pi$-radical site on the curved aromatic corannulene in Figure 1 e).

Figure $1 \mathrm{f})-\mathrm{j}$ ) shows the impact of delocalization on $\pi$-radicals, however, we still find that aromaticity drives the location of the delocalized $\pi$-radical. For indenyl [Fig. 1 f)] the Clar analysis provides one sextet with a radical in resonance between two sites, with spin density showing that the radical is indeed split, but still localized in this small species. Protonation of a benzene

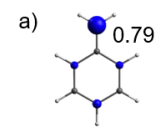

b)

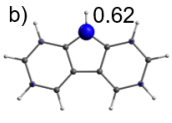

c)

d) 0.68

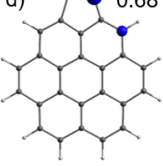

e)
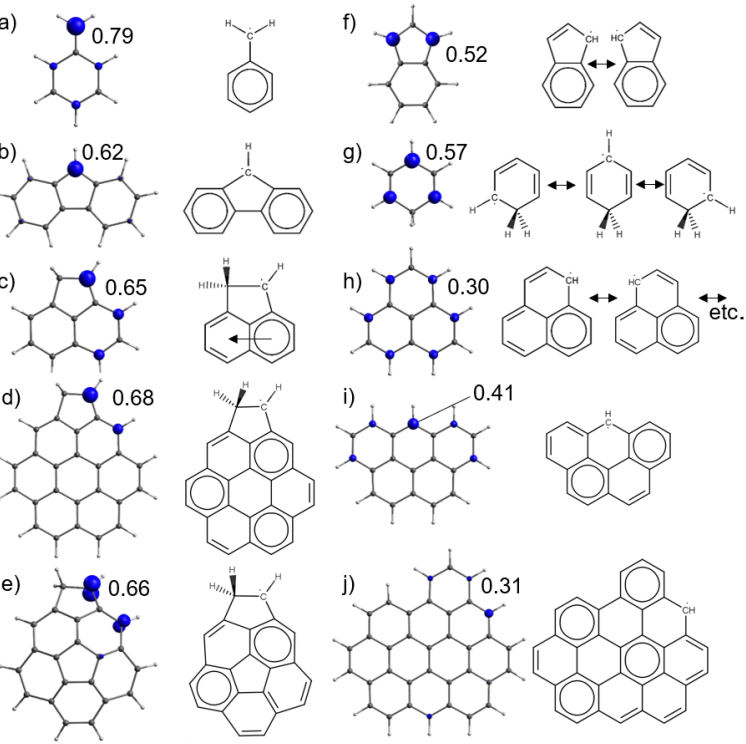

h) 0.30
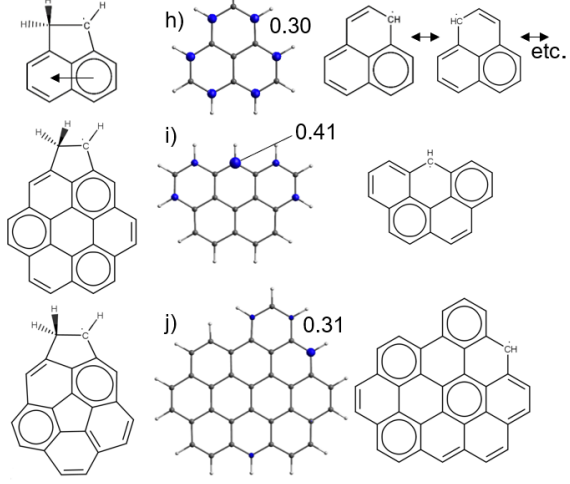

Figure 1: Spin density isosurface $=0.025$ shown on the molecular geometry (left) with the Clar analysis (right). The spin density of the most spin rich carbon site is also shown indicating the degree of localization.

ring [Fig. $1 \mathrm{~g}$ )] again suggests three locations for the $\pi$-radical beginning to become delocalized. Phenalenyl [Fig. $1 \mathrm{~h}$ )], the prototypical six-membered ring delocalized $\pi$-radical, has the radical split over six sites, still driven by aromaticity. The Clar analysis shows one sextet with the radical residing on the zig-zag edge with six resonance structures suggesting significant delocalization. Enlarging the aromatic network in this case [Fig. 1 i) and j)] leads to a delocalization of the $\pi$-radical with some minor localization on zig-zag edges, which can be justified by a major Clar structure with the radical centered on these edges. Therefore, the $\pi$-radical can be either localized or delocalized depending on the aromatic topology, which can be rationalized by aromaticity rules.

\subsection{Reaction mechanism for localized $\pi$-radicals on rim-based pentagonal rings}

Entropic considerations suggest that hydrogen addition would be unfavored at high temperatures due to 1) the reverse reaction leading to two species, and 2) the hydrogen addition reactions have a larger preexponential factor, as the additions can occur from above and below the aromatic plane, whereas hydrogen abstraction requires a collision directly along the $\mathrm{C}-\mathrm{H}$ bond in order to form the radical. At flame temperatures this paints a picture of rapid hydrogen addition and removal during soot formation, requiring a detailed 

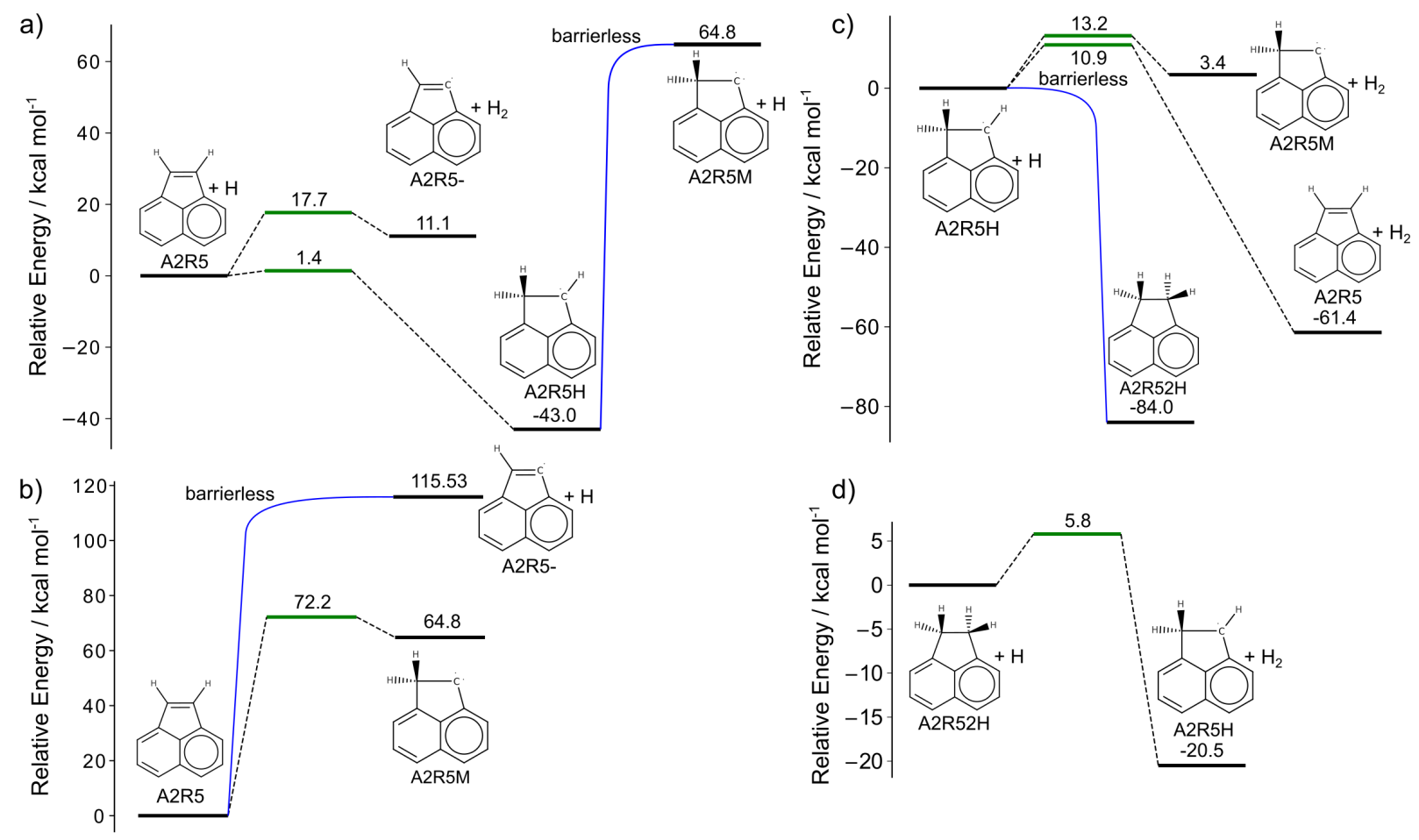

Figure 2: Potential energy surfaces for the reactions on five-member rings in acenaphthalene leading to the formation of localized $\pi$-radicals on rim-based pentagonal rings at $0 \mathrm{~K}$. Energies are computed at the CBS-QB3//B3LYP/6-311G(d,p) level of theory.

mechanism to reveal the concentration of reactive localized $\pi$-radicals on rim-based pentagonal rings. It should be mentioned that systems with biradical character are not considered due to space restrictions $[8,9]$.

Figure 2 presents the potential energy surfaces for the reactions of acenaphthalene (A2R5) that could lead to the formation of the localized $\pi$-radical A2R5H, a $\sigma$ radical (A2R5-), as well as a fully hydrogenated fivemembered ring (A2R52H). Starting from A2R5 as the reactant in Figure 2 a), A2R5- can be produced through hydrogen abstraction by an $\mathrm{H}$ radical, which proceeds with a moderate barrier of $17.7 \mathrm{kcal} / \mathrm{mol}$. This is similar to values reported for hydrogen abstraction by $H$ radicals on PAHs using the G3(MP2,CC) composite method [22]. Hydrogen can also attack the fivemembered ring on A2R5, resulting in the localized $\pi$ radical, A2R5H. This process has a very low barrier of $1.4 \mathrm{kcal} / \mathrm{mol}$, and releases $43 \mathrm{kcal} / \mathrm{mol}$ of energy, meaning it is expected to be preferred at low temperatures. Finally, A2R5H can undergo a hydrogen loss process, the reverse of the barrierless A2R5M and hydrogen radical recombination reaction. The reaction energy for the hydrogen loss in this case is high as expected, at
$108 \mathrm{kcal} / \mathrm{mol}$. In Figure 2 b), A2R5- can also be formed by a hydrogen loss from A2R5. Again, the reverse process of the A2R5- and hydrogen radical recombination is barrierless. This hydrogen loss also has a very high reaction energy of $115.5 \mathrm{kcal} / \mathrm{mol}$. A2R5 can also undergo a hydrogen migration to form A2R5M, but this requires a large barrier $(72.2 \mathrm{kcal} / \mathrm{mol})$ to be overcome in order for the migration to proceed, with a small reverse barrier. Both of these processes are expected to be unfavorable at flame temperatures [23, 24]. In Figure $2 \mathrm{c}$ ), the localized $\pi$-radical A2R5H can have hydrogen abstraction by $\mathrm{H}$ radicals occur at both carbons along the five-membered ring peripheral free edge. One abstraction results in the formation of $\mathrm{A} 2 \mathrm{R} 5 \mathrm{M}$, requiring a barrier of $13.2 \mathrm{kcal} / \mathrm{mol}$, with a reaction energy of $3.4 \mathrm{kcal} / \mathrm{mol}$. The other abstraction requires a barrier of $10.9 \mathrm{kcal} / \mathrm{mol}$ and re-produces $\mathrm{A} 2 \mathrm{R} 5$, with a reaction energy of $-61.4 \mathrm{kcal} / \mathrm{mol}$. Between the two abstraction processes, the production of A2R5 has a slightly lower barrier and proceeds to a lower energy product, so it is expected that A2R5 will be preferentially formed over A2R5M by abstraction. Alternatively, the $\mathrm{H}$ radical can undergo a barrierless recombination reaction with 
the localized $\pi$-radical A2R5H to form the protonated $\mathrm{A} 2 \mathrm{R} 52 \mathrm{H}$. With a reaction energy of $-84.0 \mathrm{kcal} / \mathrm{mol}$, this is expected to be the competing route with abstraction, producing A2R5. Finally, Figure 2 d) presents hydrogen abstraction by $\mathrm{H}$ radicals on either of the fully saturated carbon sites in A2R52H, resulting in the reformation of $\mathrm{A} 2 \mathrm{R} 5 \mathrm{H}$. This hydrogen abstraction has a much lower barrier than those seen for the abstractions on A2R5 or A2R5H, at just $5.8 \mathrm{kcal} / \mathrm{mol}$. This abstraction also proceeds downhill in energy with a decrease in $20.5 \mathrm{kcal} / \mathrm{mol}$, which is also in contrast to the A2R5 and A2R5H abstractions. This abstraction provides a return path to the localized $\pi$-radical. The rate constants for the reactions presented in the potential energy surface in Figure 2 and their reverse reactions were computed in the temperature range $300-2000 \mathrm{~K}$ and at the high pressure limit. The rate constants were then fitted to the modified Arrhenius form, with the parameters given in Table 1.

Table 1: Modified Arrhenius rate parameters for the localized $\pi$ radical mechanism. The units are $\mathrm{kcal} / \mathrm{mol}, \mathrm{K}, \mathrm{cm}^{3} \mathrm{~mol}^{-1} \mathrm{~s}^{-1}$ and $\mathrm{s}^{-1}$.

\begin{tabular}{|c|c|c|c|}
\hline Reaction & $A$ & $n$ & E \\
\hline \multicolumn{4}{|c|}{ With Barrier } \\
\hline $\mathrm{A} 2 \mathrm{R} 5+\mathrm{H} \rightarrow \mathrm{A} 2 \mathrm{R} 5-+\mathrm{H}_{2}$ & $1.99 \cdot 10^{8}$ & 1.88 & 16.76 \\
\hline $\mathrm{A} 2 \mathrm{R} 5-+\mathrm{H}_{2} \rightarrow \mathrm{A} 2 \mathrm{R} 5+\mathrm{H}$ & $7.08 \cdot 10^{5}$ & 2.11 & 4.89 \\
\hline $\mathrm{A} 2 \mathrm{R} 5+\mathrm{H} \rightarrow \mathrm{A} 2 \mathrm{R} 5 \mathrm{H}$ & $1.63 \cdot 10^{9}$ & 1.51 & 0.77 \\
\hline $\mathrm{A} 2 \mathrm{R} 5 \mathrm{H} \rightarrow \mathrm{A} 2 \mathrm{R} 5+\mathrm{H}$ & $1.93 \cdot 10^{10}$ & 1.24 & 44.66 \\
\hline $\mathrm{A} 2 \mathrm{R} 5 \rightarrow \mathrm{A} 2 \mathrm{R} 5 \mathrm{M}$ & $2.65 \cdot 10^{11}$ & 0.70 & 71.85 \\
\hline $\mathrm{A} 2 \mathrm{R} 5 \mathrm{M} \rightarrow \mathrm{A} 2 \mathrm{R} 5$ & $3.19 \cdot 10^{11}$ & 0.40 & 6.88 \\
\hline $\mathrm{A} 2 \mathrm{R} 5 \mathrm{H}+\mathrm{H} \rightarrow$ & $7.29 \cdot 10^{7}$ & 1.48 & 11.84 \\
\hline \multicolumn{4}{|l|}{$\mathrm{A} 2 \mathrm{R} 5 \mathrm{M}+\mathrm{H}_{2}$} \\
\hline $\mathrm{A} 2 \mathrm{R} 5 \mathrm{M}+\mathrm{H}_{2} \rightarrow$ & $4.85 \cdot 10^{5}$ & 1.89 & 7.49 \\
\hline \multicolumn{4}{|l|}{$\mathrm{A} 2 \mathrm{R} 5 \mathrm{H}+\mathrm{H}$} \\
\hline $\mathrm{A} 2 \mathrm{R} 5 \mathrm{H}+\mathrm{H} \rightarrow \mathrm{A} 2 \mathrm{R} 5+\mathrm{H}$ & $2.17 \cdot 10^{7}$ & 1.86 & 9.24 \\
\hline $\mathrm{A} 2 \mathrm{R} 5+\mathrm{H}_{2} \rightarrow \mathrm{A} 2 \mathrm{R} 5 \mathrm{H}+\mathrm{H}$ & $11.20 \cdot 10^{5}$ & 2.56 & 69.86 \\
\hline $\mathrm{A} 2 \mathrm{R} 52 \mathrm{H}+\mathrm{H} \rightarrow$ & $4.42 \cdot 10^{7}$ & 1.83 & 4.33 \\
\hline \multicolumn{4}{|l|}{$\mathrm{A} 2 \mathrm{R} 5 \mathrm{H}+\mathrm{H}_{2}$} \\
\hline $\mathrm{A} 2 \mathrm{R} 5 \mathrm{H}+\mathrm{H}_{2} \rightarrow$ & $2.41 \cdot 10^{6}$ & 1.78 & 24.28 \\
\hline \multicolumn{4}{|l|}{$\mathrm{A} 2 \mathrm{R} 52 \mathrm{H}+\mathrm{H}$} \\
\hline \multicolumn{4}{|c|}{ Barrierless } \\
\hline $\mathrm{A} 2 \mathrm{R} 5 \rightarrow \mathrm{A} 2 \mathrm{R} 5-+\mathrm{H}$ & $2.87 \cdot 10^{15}$ & -0.32 & 117.47 \\
\hline $\mathrm{A} 2 \mathrm{R} 5-+\mathrm{H} \rightarrow \mathrm{A} 2 \mathrm{R} 5$ & $4.90 \cdot 10^{11}$ & 0.21 & 0.00 \\
\hline $\mathrm{A} 2 \mathrm{R} 5 \mathrm{H} \rightarrow \mathrm{A} 2 \mathrm{R} 5 \mathrm{M}+\mathrm{H}$ & $4.61 \cdot 10^{15}$ & -0.35 & 109.87 \\
\hline $\mathrm{A} 2 \mathrm{R} 5 \mathrm{M}+\mathrm{H} \rightarrow \mathrm{A} 2 \mathrm{R} 5 \mathrm{H}$ & $1.19 \cdot 10^{13}$ & 0.09 & 0.28 \\
\hline $\mathrm{A} 2 \mathrm{R} 52 \mathrm{H} \rightarrow \mathrm{A} 2 \mathrm{R} 5 \mathrm{H}+\mathrm{H}$ & $1.89 \cdot 10^{16}$ & -0.40 & 86.38 \\
\hline $\mathrm{A} 2 \mathrm{R} 5 \mathrm{H}+\mathrm{H} \rightarrow \mathrm{A} 2 \mathrm{R} 52 \mathrm{H}$ & $6.95 \cdot 10^{12}$ & 0.10 & 0.35 \\
\hline
\end{tabular}

To examine the accuracy of the rate coefficients calculated above, one can compare the rate constants for the hydrogen abstraction by $\mathrm{H}$ radicals to those previ- ously calculated by the authors [22], where we calculated the rate of abstraction from A2R5. The value calculated using CBS-QB3 is higher by a factor of 1.72 at $1500 \mathrm{~K}$. This follows from the barrier for this abstraction predicted by CBS-QB3, being $1.8 \mathrm{kcal} / \mathrm{mol}$ lower than the previous calculations, which is within their reported uncertainty of $\pm 2 \mathrm{kcal} / \mathrm{mol}$ for the M06- $2 \mathrm{X}$ barrier heights. Additionally, Violi reported a barrier of $80 \mathrm{kcal} / \mathrm{mol}$ for migration of a hydrogen atom on the bay site of benzo[c]phenanthrene, resulting in the formation of a carbene site [23]. This is not too dissimilar to the migration barrier of $72.2 \mathrm{kcal} / \mathrm{mol}$ for $\mathrm{A} 2 \mathrm{R} 5$ in this work, given that the results from [23] were computed using energies at B3LYP/6-31G(d,p) compared to CBSQB3. The barrier heights were also computed with the G4MP2 method and M06-2X functional,with the mean deviation in barrier heights being 1.1 and $2.3 \mathrm{kcal} / \mathrm{mol}$, respectively (see supplemental information). This suggests reasonable agreement with other methods.

For the barrierless recombination reactions, Mebel and coworkers computed the reaction energy of the $\mathrm{H}$ radical-naphthyl recombination as $-111.0 \mathrm{kcal} / \mathrm{mol}$ using G3(MP2,CC) [25]. This reaction is similar to the $\mathrm{H}$ radical-A2R5- recombination, as is our energy of $-115.5 \mathrm{kcal} / \mathrm{mol}$. The reaction energy of A2R5M and $\mathrm{H}$ is also similar at $-107.8 \mathrm{kcal} / \mathrm{mol}$. The $\mathrm{A} 2 \mathrm{R} 5 \mathrm{H}$ recombination reaction energy is quite different at $84.0 \mathrm{kcal} / \mathrm{mol}$, but the $\mathrm{H}$ radical attacks a carbon that already has a hydrogen, which is not true for the other reactions, so this difference is expected. Harding et al. computed the rate of the $\mathrm{H}$ radical-naphthyl recombination reaction using interaction energies determined by CASPT2/cc-pVDZ and VRC-TST for the rates [26]. In general, the rate constants calculated by Harding for the $\mathrm{H}$ radical-naphthyl recombination are one to two orders of magnitude higher than the recombinations here. The calculations here are less accurate, as the interaction energies are determined by DFT and not a multi-reference method. On the other hand, it was noted that for hydrogen abstraction reactions, the reaction on A2R5 is several orders of magnitude lower than for naphthyl [22], so this difference may be due to the reactant molecule. Additionally, the rate of the $\mathrm{H}$ radical-A2R5- recombination was also computed using $\operatorname{CASSCF}(2 \mathrm{e}, 2 \mathrm{o})$ interaction energies (see supplemental information), with the predicted rate constant being 6-11\% lower, suggesting B3LYP agrees reasonably with higher level methods. Nevertheless, further refinement of the rates of the barrierless reactions is necessary, as these will be the largest source of uncertainty in the kinetic mechanism. 

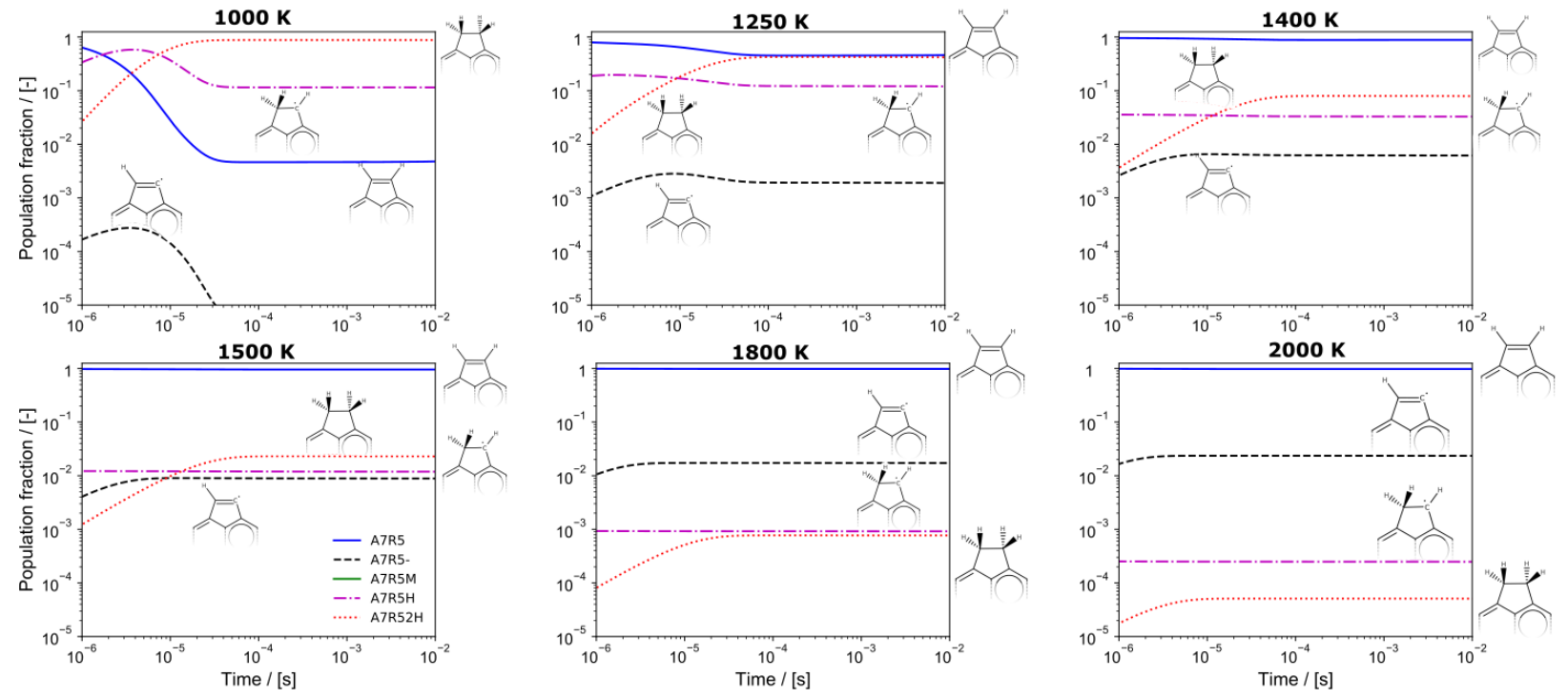

Figure 3: 0-dimensional homogeneous gas-phase reactor simulations of reactions involving acecoronene (A7R5) at atmospheric pressure and typical flame compositions. The degree of saturation of the five-membered ring is shown in the figure.

\subsection{Concentration profiles in flames}

Figure 3 provides the kinetic simulations in a 0 $\mathrm{D}$, homogeneous gas-phase reactor at 1 atmosphere of pressure using the 18 reactions that involve the fivemembered ring detailed in Table 1 . The chosen reactant PAH is acecoronene, a coronene with a rimbased five-membered ring or A7R5, representative of a slightly larger PAH. Since reactions of carbons in the six-membered ring are not considered (as the fivemembered ring is of primary interest), the choice of reactant PAH is largely unimportant. The rates computed for A2R5 are also likely very similar for larger PAHs, which has been observed for hydrogen abstraction by $\mathrm{H}$ radicals on PAHs [22]. Computations we performed of rate constants for hydrogen abstraction, migration, and addition for A7R5 suggest this is indeed the case (see Supplementary information). The kinetic simulations were conducted for six temperatures, including a lower temperature threshold for soot formation of $>1000 \mathrm{~K}$, temperatures of 1250 and $1400 \mathrm{~K}$ where soot nucleation is found in flames [27], a typical flame temperature of $1500 \mathrm{~K}$, and temperatures representative of maximum values seen in flames of 1800 and $2000 \mathrm{~K}$.

Figure 3 shows clear trends in the degree of saturation of the carbons on the five-membered ring. At $1000 \mathrm{~K}$, the saturated products are preferred, with the fully saturated species being the dominant product at a population fraction (mole fraction divided by total PAH mole fraction) close to one. In other words, most of the reactant PAH transforms to a fully saturated five-membered ring product. However, the localized $\pi$-radical is the second most favored product with population fraction close to 0.1 . The reactant PAH is depleted, but then starts to reform at around $1 \mathrm{~ms}$ when the reverse reactions start to occur. At 1250 and $1400 \mathrm{~K}$, it can be observed that the major product is the reactant with an unsaturated rim-based pentagonal ring, followed by the fully saturated five-membered ring and the localized $\pi$ radical. Comparing these results with the HR-AFM structures we find a consistent frequency of rim-based pentagonal sites with a ratio of 27:12:4 for the unsaturated, saturated and partially saturated rim-based pentagonal rings [4]. The population fraction of the saturated product is lower at these temperatures, but is still of the order of 1 to 0.1 for the fully saturated fivemembered ring and 0.1 to 0.01 for the localized $\pi$ radical. This is a significant population fraction and is considerably higher than the $\sigma$-radicals, suggesting that if chemical cross-linking reactions leading to larger PAHs and/or soot are to occur at these temperatures, such reactions should also consider localized $\pi$-radicals as potential reactants. At $1500 \mathrm{~K}$, the population fraction of the localized $\pi$-radical is stable at 0.01 , with the population fraction of $\sigma$-radicals being very similar and the population fraction of the fully saturated product being only slightly higher. This suggests that even at $1500 \mathrm{~K}$, the localized $\pi$-radicals could be as important as $\sigma$-radicals, even though their concentration is much lower than just the species with peripheral fivemembered rings. At the higher temperatures of 1800 
and $2000 \mathrm{~K}$, the unsaturated states are favored, with the reactant and $\sigma$-radicals being the dominant species as consistent with the trend. However, it can be seen that the population fraction of $\sigma$-radicals at these temperatures is lower than what was observed for the $\pi$ radicals at lower temperatures. A sensitivity analysis of the population fractions to the computed rate constants was conducted (see supplemental information), identifying the $\mathrm{R} 5+\mathrm{H}$ reaction as the most important reaction. Additionally, simulations using initial $\mathrm{H}$ mole fractions of $10^{-4}$ and 0.01 were also conducted (see supplemental information), showing that the fraction of initial PAH converted to the localized $\pi$-radical, fully saturated product, and $\sigma$-radical is proportional to this initial $\mathrm{H}$ mole fraction, but the relative importance of these species remains unchanged. These two analyses suggest that the localized $\pi$-radicals are important given sufficient $\mathrm{H}$-atom concentration.

Finally, a kinetic Monte Carlo scheme was developed to see if multiple localized $\pi$-radicals could be present on a single PAH with multiple rim-based pentagons (Figure 4). The lowest temperature (1000 K) was used to study the localized $\pi$-radicals as the kinetic simulations suggest that they are present in higher concentrations. , The population fraction of PAHs with one localized $\pi$-radical reaches a maximum of $\sim 0.5$ at around $10^{-6} \mathrm{~s}$ for both the two and three rim-based pentagonal ring cases. These PAHs subsequently react to form those with multiple localized $\pi$-radicals. In the two-site case, the two $\pi$-radical PAHs reach a significant population fraction of 0.35 , whilst in the three-site case, the two and three $\pi$-radical PAHs of 0.4 and 0.2 , respectively. At this point, a majority of PAHs have multiple localized $\pi$-radical sites. From here, the fully saturated product becomes dominant but in both cases a significant fraction of PAHs with single site localized $\pi$ radical remains. At very long timescales, the fully saturated product starts to reform the localized $\pi$-radical due to reversibility.

Comparing these results with the frequency of aromatic species imaged with HR-AFM [4] just prior to soot nucleation, from a total of 49 molecules with $>4$ rings; 28 contained at least one rim-based pentagonal ring, 12 contained two rim-based pentagonal rings and none of the imaged species contain three rim-based pentagonal rings. At least two reactive sites would be required to allow for a continuous mass accumulation into soot nanoparticles given a mechanism only involving ARLH. It should be mentioned that we also found that the localized $\pi$-radical is able to form strong $\pi$-stacked complexes with the partially embedded rim-based pentagonal [6]. However, computing the concentrations of this site is outside the scope of this paper, as it requires detailed chemistry of PAH growth. The relative importance of $\pi$-radical versus $\sigma$-radical was also not explored due to the challenge of including van der Waals and possible curved PAH flexoelectric driven interactions for $\pi$-radicals in ARLH, which could significantly enhance the efficiency of reactions during collisions with flexoelectric species, which has been one of the challenges in $\sigma$-driven mechanisms [28]. Given these considerations it appears, from these preliminary results, that $\pi$-radicals on rim-based pentagonal rings have sufficient concentrations and reactivity to be relevant to $\mathrm{PAH}$ and soot precursor chemistry.

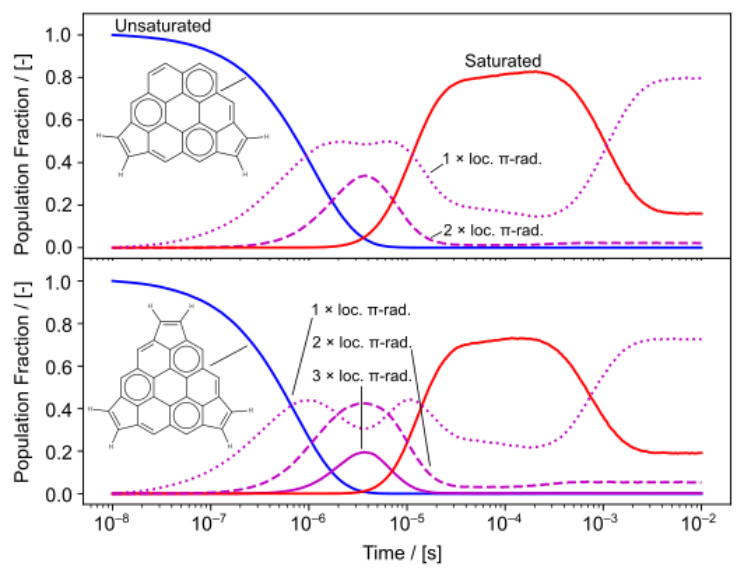

Figure 4: Kinetic Monte Carlo simulation for the time evolution of rim-based pentagonal rings for two (top) and three (bottom) site species at atmospheric pressure and typical flame compositions at $1000 \mathrm{~K}$. These results are the average of five runs.

\section{Conclusions}

Localization of $\pi$-radicals was found to be governed by aromaticity rules developed from a Clar analysis. The partially saturated rim-based pentagonal ring was found to concentrate significant spin density on the pentagonal ring and be unchanged by extension of the aromatic ring, as opposed to $\pi$-radicals formed within the six-membered ring networks which delocalize. A reaction mechanism was developed, allowing the concentration of possible localized $\pi$-radicals to be computed within flame conditions with significant concentrations found in soot-forming temperatures provided sufficient H-radical concentrations are present. Finally, kinetic Monte Carlo simulations showed that multiple reactive sites can be formed on a single aromatic species, suggesting a chemical polymerization could be possible. This will require further computational and experimental work to establish. 


\section{Acknowledgments}

This project is supported by the National Research Foundation (NRF), Prime Minister's Office, Singapore under its Campus for Research Excellence and Technological Enterprise (CREATE) programme. AM gratefully acknowledges Johnson Matthey for financial support. GL is funded by a CONACYT Cambridge Scholarship and wishes to acknowledge both institutions, the National Council of Science and Technology and the Cambridge Commonwealth Trust. MK gratefully acknowledges the support of the Alexander von Humboldt foundation.

\section{References}

[1] P. J. Landrigan, R. Fuller, N. J. R. Acosta, O. Adeyi, R. Arnold, N. N. Basu, A. B. Baldé, R. Bertollini, S. Bose-O'Reilly, J. I. Boufford, P. N. Breysse, T. Chiles, et al., The Lancet Commission on pollution and health, The Lancet (2017).

[2] J. R. McConnell, R. Edwards, G. L. Kok, M. G. Flanner, C. S. Zender, E. S. Saltzman, J. R. Banta, D. R. Pasteris, M. M. Carter, J. D. W. Kahl, 20th-Century Industrial Black Carbon Emissions Altered Arctic Climate Forcing, Science 317 (2007) 1381-1384.

[3] T. C. Bond, S. J. Doherty, D. W. Fahey, P. M. Forster, T. Berntsen, B. J. Deangelo, M. G. Flanner, S. Ghan, B. Kärcher, D. Koch, et al., Bounding the role of black carbon in the climate system: A scientific assessment, J. Geophys. Res.:Atmospheres 118 (2013) 5380-5552.

[4] M. Commodo, K. Kaiser, G. De Falco, P. Minutolo, F. Schulz, A. D'Anna, L. Gross, On the early stages of soot formation: Molecular structure elucidation by high-resolution atomic force microscopy, Combust. Flame 205 (2019) 154-164.

[5] K. Johansson, M. Head-Gordon, P. Schrader, K. Wilson, H. Michelsen, Resonance-stabilized hydrocarbon-radical chain reactions may explain soot inception and growth, Science 361 (2018) 997-1000.

[6] J. W. Martin, D. Hou, A. Menon, J. Akroyd, X. You, M. Kraft, Reactivity of polycyclic aromatic hydrocarbon radicals: implications for soot formation, J. Phys. Chem. C 123 (2019) 26673.

[7] R. Whitesides, M. Frenklach, Detailed kinetic Monte Carlo simulations of graphene-edge growth., J. Phys. Chem. A 114 (2010) 689-703.

[8] H. Wang, Formation of nascent soot and other condensed-phase materials in flames, P. Combust. Inst. 33 (2011) $41-67$.

[9] D. Koley, E. Arunan, S. Ramakrishnan, Computational investigations on covalent dimerization/oligomerization of polyacenes: Is it relevant to soot formation?, J. Comp. Chem. 33 (2012) 1762-1772.

[10] J. B. Howard, Carbon addition and oxidation reactions in heterogeneous combustion and soot formation, Symp. (Int.) Combust., [Proc.] 23 (1991) 1107-1127.

[11] M. Frisch, G. Trucks, H. Schlegel, G. Scuseria, M. Robb, J. Cheeseman, G. Scalmani, V. Barone, G. Petersson, H. Nakatsuji, et al., Gaussian 16, Revision A 3 (2016).

[12] C. W. Gao, J. W. Allen, W. H. Green, R. H. West, Reaction mechanism generator: Automatic construction of chemical kinetic mechanisms, Comput. Phys. Commun. 203 (2016) 212225.

[13] E. P. Wigner, On the quantum correction for thermodynamic equilibrium, in: Part I: Physical Chemistry. Part II: Solid State Physics, Springer, 1997, pp. 110-120.

[14] K. Rynefors, Unimol: A program for monte carlo simulation of rrkm unimolecular decomposition in molecular beam experiments, Comp. Phys. Commun. 27 (1982) 201-212.

[15] D. G. Truhlar, B. C. Garrett, Variational transition state theory, Annu. Rev. Phys. Chem. 35 (1984) 159-189.

[16] J. Barker, T. Nguyen, J. Stanton, C. Aieta, M. Ceotto, F. Gabas, T. Kumar, C. Li, L. Lohr, A. Maranzana, et al., Multiwell-2017 software suite, Ann Arbor, Michigan (2017).

[17] M. Akbar Ali, J. R. Barker, Comparison of three isoelectronic multiple-well reaction systems: $\mathrm{OH}+\mathrm{CH}_{2} \mathrm{O}, \mathrm{OH}+\mathrm{CH}_{2} \mathrm{CH}_{2}$, and $\mathrm{OH}+\mathrm{CH}_{2} \mathrm{NH}$, J. Phys. Chem. A. 119 (2015) 7578-7592.

[18] D. G. Goodwin, H. K. Moffat, R. L. Speth, Cantera: An objectoriented software toolkit for chemical kinetics, thermodynamics, and transport processes, Caltech, Pasadena, CA (2009).

[19] A. Raj, Structural effects on the growth of large polycyclic aromatic hydrocarbons by c2h2, Combust. Flame 204 (2019) 331340.

[20] M. Solà, Forty years of clar's aromatic $\pi$-sextet rule, Front. Chem. 1 (2013) 22.

[21] M. Liu, W. H. Green, Capturing aromaticity in automatic mechanism generation software, Proc. Combust. Inst. 37 (2019) 575581.

[22] D. Hou, X. You, Reaction kinetics of hydrogen abstraction from polycyclic aromatic hydrocarbons by h atoms, Phys. Chem. Chem. Phys. 19 (2017) 30772-30780.

[23] A. Violi, Cyclodehydrogenation reactions to cyclopentafused polycyclic aromatic hydrocarbons, J. Phys. Chem A. 109 (2005) 7781-7787.

[24] M. Frenklach, R. I. Singh, A. M. Mebel, On the low-temperature limit of haca, Proc. Combust. Inst. 37 (2019) 969-976.

[25] A. Mebel, V. Kislov, Can the c5h5+c5h5 $\rightarrow \mathrm{c} 10 \mathrm{~h} 10 \rightarrow \mathrm{c} 10 \mathrm{~h} 9+$ $\mathrm{h} / \mathrm{c} 10 \mathrm{~h} 8+\mathrm{h} 2$ reaction produce naphthalene? an ab initio/rrkm study, J. Phys. Chem. A 113 (2009) 9825-9833.

[26] L. B. Harding, Y. Georgievskii, S. J. Klippenstein, Predictive theory for hydrogen atom- hydrocarbon radical association kinetics, J. Phys. Chem. A 109 (2005) 4646-4656.

[27] I. Glassman, Soot formation in combustion processes, Symposium (International) on Combustion 22 (1989) 295-311.

[28] Q. Mao, D. Hou, K. H. Luo, X. You, Dimerization of polycyclic aromatic hydrocarbon molecules and radicals under flame conditions, J. Phys. Chem A. 122 (2018) 8701-8708. 


\section{Supplementary information}

The supplementary information for "Reactive localized $\pi$-radical aromatic soot precursors: properties and concentration in flames" includes comparisons of quantum chemical methods, further details on the rate calculations presented in the main paper, further details on spin density analysis, a sensitivity analysis on the results of the kinetic simulations, a full description of the KMC model, and the molecular parameters used in this work. This includes:

- Table S1: Comparison of barrier heights predicted by CBS-QB3, G4MP2, and M06-2X methods. The units are $\mathrm{kcal} / \mathrm{mol}$.

- A comparison of the rates of hydrogen abstraction, addition, and migration for A2R5 and A7R5.

- Figure S1: Temperature dependent rate constants for acenapthelene (A2R5) and acecoronene (A7R5) for some critical reactions in the reaction mechanism.

- Details and comparisons of the rates of the barrierless radical recombination reactions computed by the RRKM/Gorin Model method and VTST methods.

- Figure S2: Fitted Morse potentials for barrierless reactions. The interaction potential energy surfaces were fitted to the B3LYP/6-311G(d,p) level of theory.

- Figure S3: Comparison between rate constant computed for the A2R5- + H radical recombination reaction using B3LYP and $\operatorname{CASSCF}(2 \mathrm{e}, 2 \mathrm{o})$.

- Figure S4: Temperature dependent rate constant for the three barrierless reactions computed using the Gorin model and variational TST (VTST).

- Figure S5: Kinetic simulations of a 0D homogeneous reactor with varying initial mole fractions of atomic hydrogen at temperatures of $1400 \mathrm{~K}$ and $1500 \mathrm{~K}$.

- Figure S6: Normalised sensitivity coefficients for the main PAHs to the rate constants in the kinetic mechanism.

- Table S2: Reactions used in the kinetic Monte Carlo simulations and their rate parameters

- Table S3: Reactions used in the kinetic Monte Carlo simulations and their rate parameters used in this work 